\title{
A MODELING APPROACH FOR ELASTIC TOLERANCE SIMULATION OF THE BODY IN WHITE HANG-ON PARTS
}

\author{
Zheng, Hanchen (1); Litwa, Frank (1); Reese, Benjamin (2); Li, Chenyang (2); Bohn, Martin (1); \\ Paetzold, Kristin (3)
}

1: Daimler AG; 2: Dimensional Control Systems Inc.; 3: Universität der Bundeswehr München

\begin{abstract}
Computer aided tolerancing (CAT) in the automobile industry is implemented by CAD tools. These tools analyze the manufacturability of complex assemblies with rigid single parts in an early stage to reduce the product development time and the cost for hardware prototypes. This paper proposes an approach to implement tolerance simulation for a compliant assembly, which includes manufacturing processes such as clinching, bolting and hemming by applying tolerance simulation tool. The fenderBIW system is simulated as a compliant-rigid system and the simulation model is applied to two production scenarios. The simulation results are compared with real measurement data, which demonstrates the efficacy of using simulation in early production as opposed to prototyping or other methods of design by showing the strong correlation between simulation results and as-built products.
\end{abstract}

Keywords: Tolerance representation and management, Elastic tolerance simulation, Design for $\mathrm{X}$ (DfX), Simulation, Compliant assembly

\section{Contact:}

Zheng, Hanchen

Daimler AG

TF/VDF

Germany

hanchen.zheng@daimler.com 


\section{INTRODUCTION}

\subsection{Tolerance analysis in automobile processes}

Currently, computer aided tolerancing (CAT) in the automobile industry is implemented by digital tools. These tools analyze the manufacturability of complex assemblies in an early stage to reduce the product development time and the cost for hardware prototypes. Camelio and $\mathrm{Hu}$ (2003) summarized the propagation of variation analysis models from single station to multi-station for rigid parts. He also proposed a modeling methodology to simulate the multi-station assembly process of a compliant sidewall of automobile. Schleich and Wartzack (2016) summarized the major approaches applied regarding the rigid mechanical assemblies. Corrado and Polini (2017) presented a general method to integrate the manufacturing signature and the assembly conditions into the existing theoretical models of tolerance analysis for rigid parts. At the same time, the finite element method (FEM) was also applied in the tolerance analysis. For example, $\mathrm{Liu}$ and $\mathrm{Hu}$ (1997) developed the method of influence coefficient (MIC) to simulate compliant sheet metal assemblies, Chang and Grssard (1997) proposed PCFR (Positioning, Clamping, Fastening and Releasing) cycle to model the assembly of compliant parts, butt joint and a slip joint in a body in white (BIW) process are analyzed in the work of Moos and Vezzetti (2012).

However, the conventional statistical tolerance simulation results has a shortage in fitting to the real manufacturing processes. The rigid tolerance simulation ignores any deformation in the parts, which can be caused by clamping, welding, clinching, riveting or other over-constraining operations. FEM simulation is capable of simulating the compliant part, but the simulation model must be modified for each case and the length of the simulation run time scales with model size and complexity.

\subsection{Tolerance simulation for BIW hang-on parts}

During the assembly process of the BIW, the hang-on parts should fulfill the optical functions as well as the technical functions, the requirements of which are documented in the gap and radii plan (Bohn and Hetsch, 2013). To predict the consequences of geometry variants on product quality, simplify the assembling process, and reduce the cost, tolerance simulation models are widely applied to analyze the manufacturability of complex assemblies under a series of production conditions. Ding et al. (2000) characterized variation propagation in a multi-station manufacturing process of rigid parts using space state model, Yan et al. (2015) applied Monte Carlo method (MCM) to study geometric tolerance of rigid assembly due to manufacturing processes.

The increasing complexity of the BIW assembly chain leads to a higher demand for simulation accuracy of assembly processes. To optimize the reliability of tolerance simulation for BIW hang-on parts, the influence of manufacturing processes needs to be considered. Based on linear assumption and, Gerbino et al. (2008) and Franciosa (2009) applied SVA-FEA method to statistically simulate both single- and multi-station assembly processes of compliant parts by deviating the parts with MCM and calculating the global sensitivity matrix for each station with FEM. Modern tolerance analysis tools, e.g. VisVSA FEA Module (Siemens) and 3DCS FEA Compliant Modeler (Dimensional Control System, 2008-2016), already offer possibilities to cover the shortcoming of conventional tolerance simulation approach by combining FEM information during the modeling process, called Elastic Tolerance Simulation. Previously, Chen (2014) used 3DCS to simulate the deviation of components due to the tolerance between pin locator and hole in sheet metal assembly, Wang (2018) presented a tolerance simulation of composite elevator assembly involving clamping force along with VisVSA, but the assembly processes such as clinching, bolting, and hemming, were not considered.

Table 1. Overview of the recent work of tolerance simulation involving manufacturing effects

\begin{tabular}{|l|c|c|}
\hline Numerically verified/validated & Rigid assembly & Compliant assembly \\
\hline Ding et al. (2000); & $\begin{array}{c}\text { Yan } \text { et } \text { (2015); } \\
\text { Corrado and Polini (2017); }\end{array}$ & $\begin{array}{c}\text { Camelio and Hu (2003); } \\
\text { Franciosa (2009); }\end{array}$ \\
\hline Experimentally validated & $\begin{array}{c}\text { Barbero (2014); } \\
\text { Beckmann } \text { et al. (2015); }\end{array}$ & In this paper \\
\hline
\end{tabular}


Table 1 is an overview of the recent work concerning tolerance simulation involving real manufacturing effects. Comparatively, little research has been done to validate the tolerance simulation using real measurement data. Barbero (2014) validated the tolerance simulation for an automobile locking device by comparing the measurement for the position of rivet pin axis in the hole, Beckmann et al. (2015) compared the deviations of trunk lid based on real measurements and rigid tolerance simulation. Therefore, the target of this paper is to create an elastic tolerance simulation model for an automotive fender taking into consideration multi-station manufacturing processes (e.g. clinching, hemming, bolting). The part variation comparing to its $\mathrm{CAD}$ zero position is measured in the simulation. The simulation results are then validated by comparing them to the actual as-built measurement data.

\section{TOLERANCE MODELING CONSIDERING MANUFACTURING PROCESSES}

The assembly process of BIW hang-on parts is a multi-station process (including clinching process and hemming process). Both mentioned CAT tools provide the ability to simulate the compliant assembly process virtually. 3DCS FEA Compliant Modeler is developed mainly based on the method proposed by Camelio (2004). For a sheet metal assembly system with geometric covariance and large number of variation sources, it substantially reduces the computational effort comparing to VisVSA (developed based on MIC). Therefore, the elastic tolerance simulation in this paper is implemented by using 3DCS FEA Compliant Modeler. An approach to implement the elastic tolerance modeling using 3DCS is introduced in Figure 1. The CAD solid geometry of the assembly (exported from NX) can be imported into 3DCS with the DCS converter. After importing, DCS points can be defined by typing in the coordinates and normal vector, or directly inserting at the geometry surface to create a point-based rigid simulation model. Tolerances and measurements are defined according to the user's specification. After combining the FEM information with the tolerance model, many manufacturing processes can be simulated by utilizing the compliant moves according to the assembly sequence. The general procedures for using 3DCS are specified in the user guidelines (Dimensional Control System, 2008), but the modeling approach needs to be adjusted for specific simulation systems.

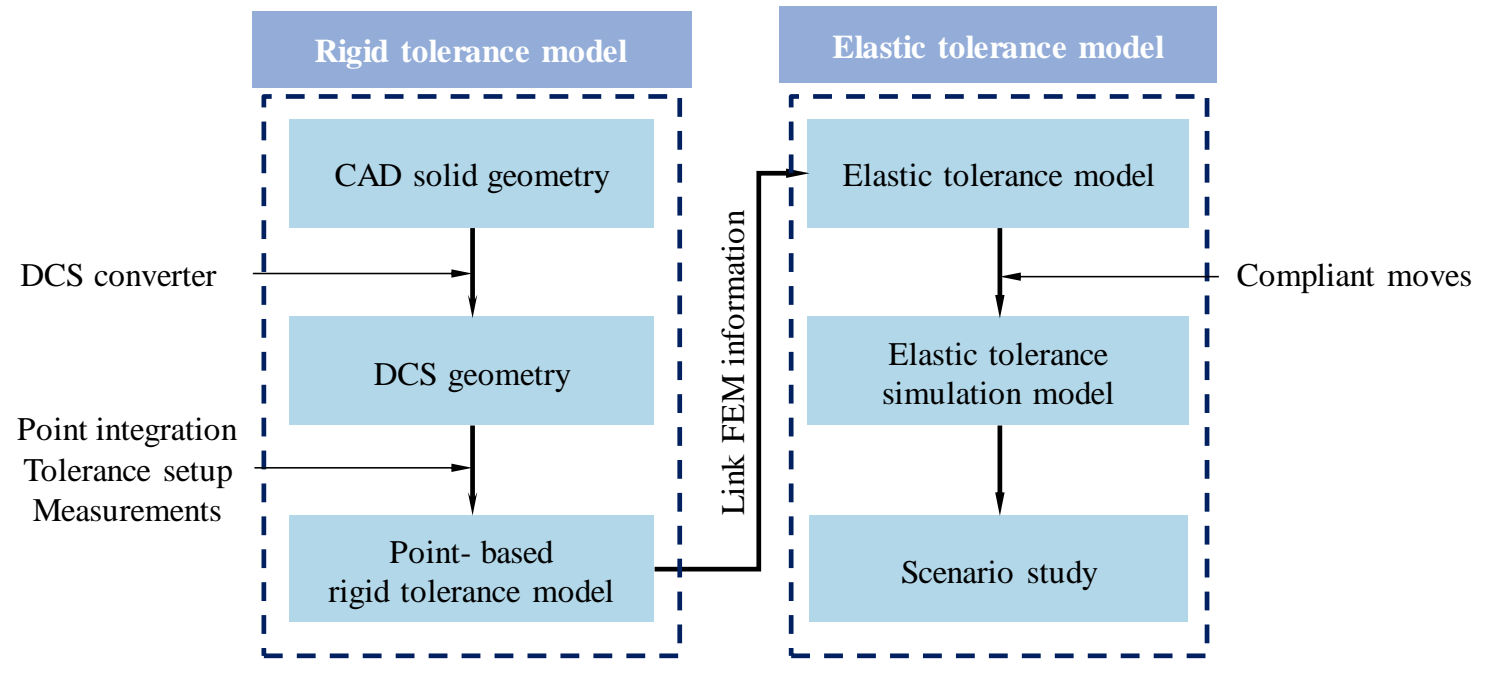

Figure 1: Approach to construct elastic tolerance model

\section{APPLICATION IN THE ASSEMBLY PROCESS OF THE FENDER-BIW SYSTEM}

The global car coordinate system (Bohn and Hetsch, 2013) is used to locate the BIW parts, in which the positive axial directions are defined refer to the driving direction: $+\mathrm{X}$ points the rear, $+\mathrm{Y}$ points the right, $+\mathrm{Z}$ is upwards (Figure 5). In the installation process of the fender to the BIW, deviation in -X direction occurs at the bottom of the fender due to manufacturing error. This deviation will cause a deformation of the whole fender assembly, concentrated at the front of the part. In order to verify this phenomenon, a simulation model of the fender-BIW system was created using the elastic tolerance simulation approach. 


\subsection{Tolerance modeling for the fender assembly}
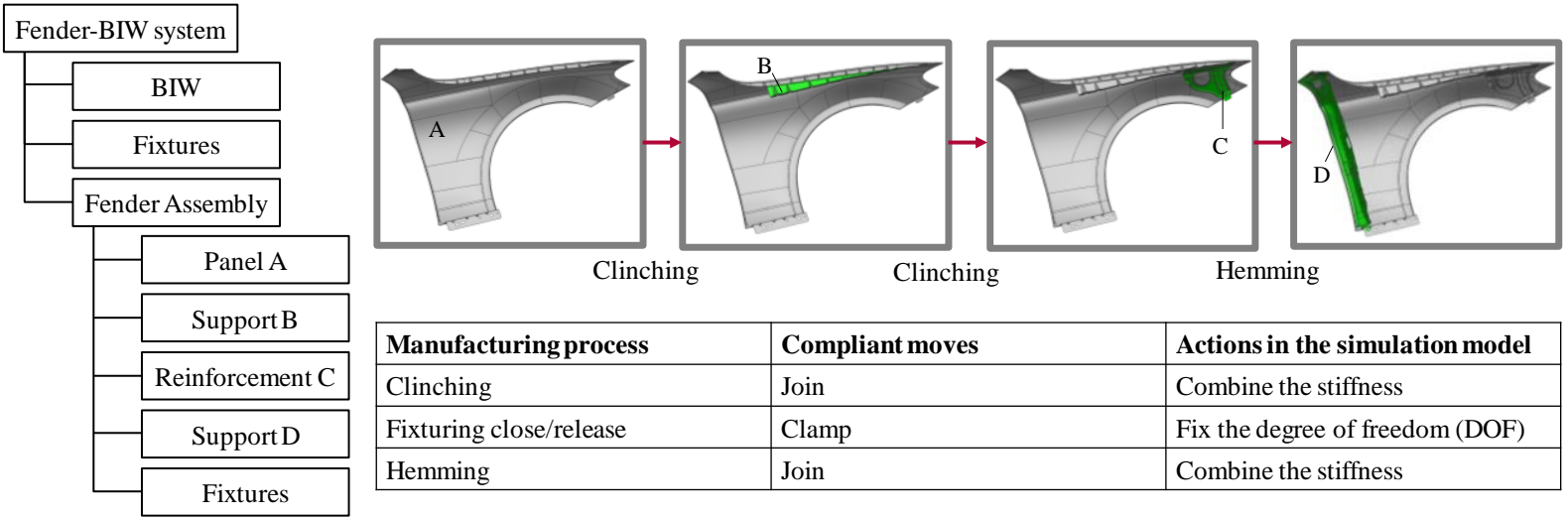

Figure 2: Overview of the assembly system

Figure 2 is an overview of the fender-BIW system. The fender assembly consists of four compliant components: a panel (A), two supports (B and D) and a reinforcement (C). Because the elastic behavior of the fender assembly due to manufacturing processes is studied in this paper, the deformations of the BIW and the fixtures are neglected as a simplification of the system. Therefore, the BIW as well as the fixtures in the system are assumed as a rigid "Black box", which deviates at the joining positions with the fender assembly according to tolerances specification. Following are the modeling procedures for the manufacturing process of the fender:

1. Integrate points

DCS points are the foundation of the point-based simulation model. For the compliant fender assembly, DCS points are created on the surfaces, which contain tolerances, manufacturing actions or measurement points. For the rigid BIW and fixtures, DCS points are created at the center of the fixtures or bolting positions. In this paper, the coordinates as well as the normal vectors of required DCS points are read from the 3D Master model manually and imported into tolerance simulation model through Excel.

To measure the deviation of the fender from its CAD 0 position, duplicate DCS points are created at the same position of the measurement points without any tolerance. Point-Point measurements are define to measure the distance projection on global $\mathrm{X}, \mathrm{Y}$ and $\mathrm{Z}$ directions between the dual measurement points to describe the deviation. The positions of the measurement points are shown in Figure 8.

2. Specify tolerances

The tolerances used in the simulation model are specified according to the tolerance specification given by Daimler AG for the specific parts (Figure 3) which based on Reference-Point-System (RPS). The difference between Daimler tolerance specification and ISO tolerancing standard are discussed in the work of Yan (2019). Linear tolerances are defined on the corresponding DCS points referring to their references. Circle tolerances are defined at all pin-hole joints and bolting positions to simulate position tolerances as well as size tolerances. The tolerances for fixtures are defined at the DCS points that are fixed on the fixtures. Normal distribution is selected for all tolerances.
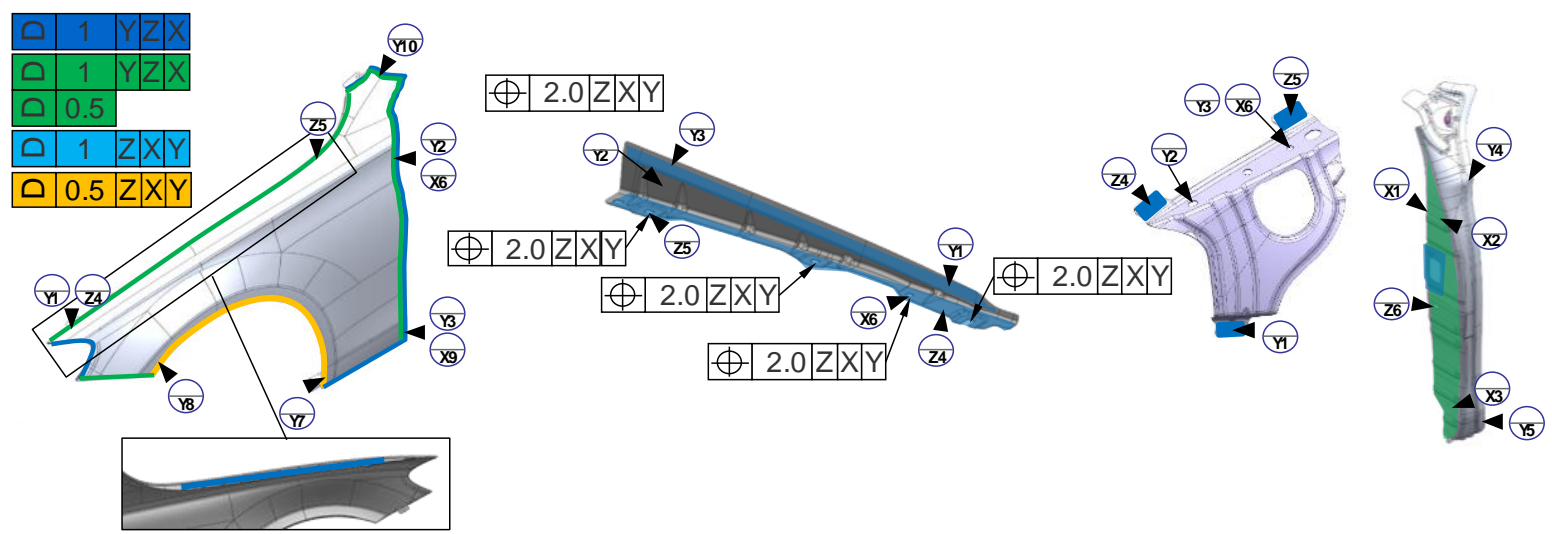

Figure 3: Tolerance specifications for single parts in fender assembly 


\section{Link FEM information}

The rigid-body tolerance model becomes an elastic tolerance model when the FEM information is loaded. In this model, all parts are made of aluminum with a thickness of $1 \mathrm{~mm}$. The material information is listed in Figure 4.

Figure 4 also indicates the process of linking FEM data to the tolerance simulation model. The DCS points on the fender are integrated with the FEM-mesh. The nodes nearest to the DCS points are grouped in the FEM model. The reduced stiffness matrix file for every compliant part is generated with the FEA tool. After the new FEM-mesh and reduced stiffness matrix are generated, the function "Load FEA data" in 3DCS Compliant Modeler links them to the simulation model so that the rigid model becomes compliant.

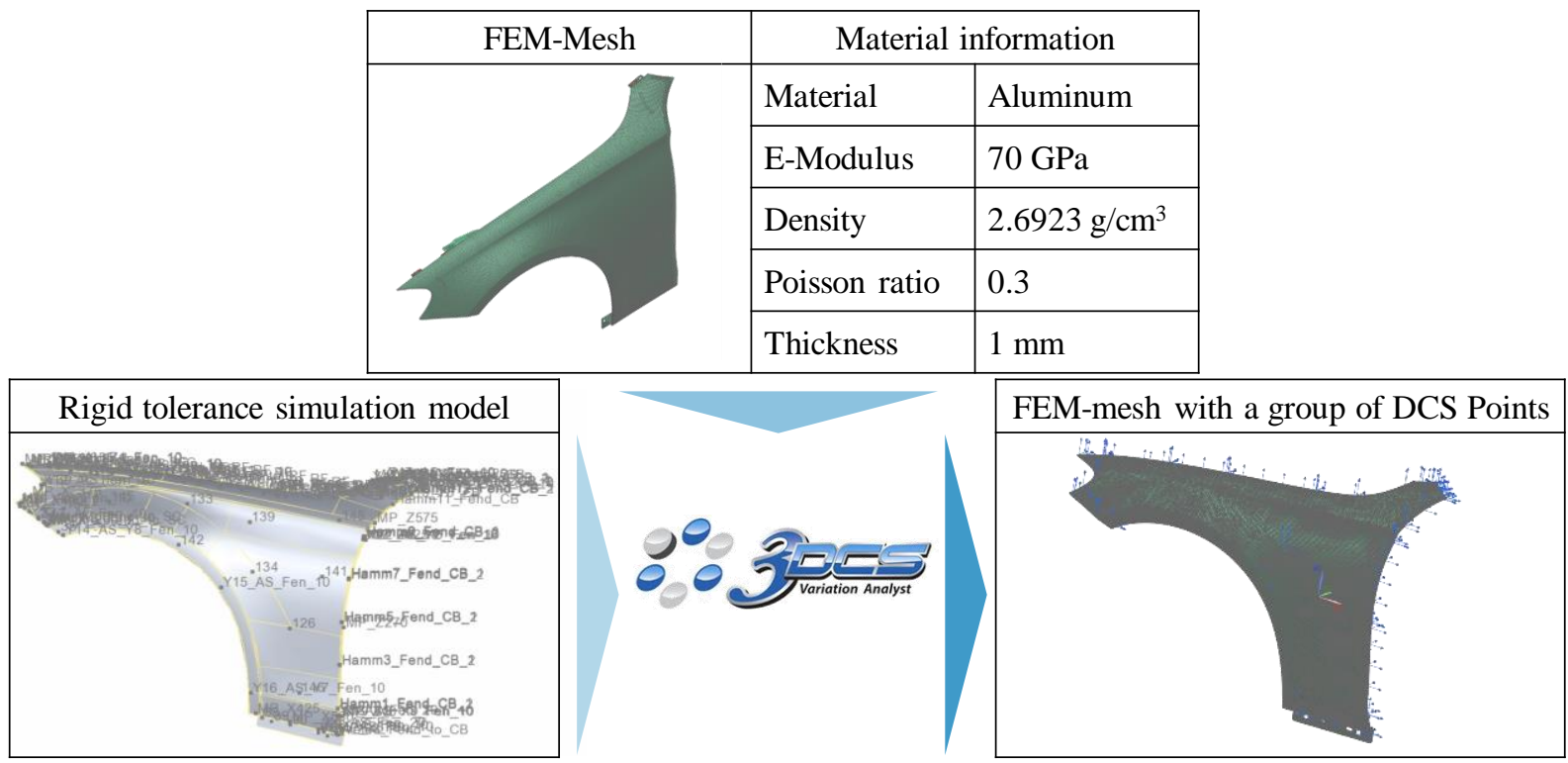

Figure 4: Link FEM information to the tolerance simulation model

4. Define manufacturing processes

The manufacturing processes of the fender consist of two stations. The first station assembles the individual components A, B, C and D together. As shown in Figure 2, the panel A and the support B are fixed on the fixtures before they are clinched together. The subassembly $\mathrm{AB}$ is released and shifted to the next joint process. The subassembly $\mathrm{AB}$ and the reinforcement $\mathrm{C}$ are then fixed on fixtures and clinched together. Finally, the support $\mathrm{D}$ is fixed at the proper position and hemmed with the subassembly $\mathrm{ABC}$ to form the fender assembly. During this joint process, the flanges of $\mathrm{A}$ and $\mathrm{D}$ are assumed to be firmly connected; the non-linear effect due to the glue between parts is not considered in this paper, so no relative sliding occurs at the contact interfaces during the hemming process. Finally, the fender assembly is released from the fixtures and shifted to the next station for the mounting process.

At the second station, the fender assembly is mounted to the BIW at the positions shown in scenario 2, Figure 5. Because the BIW is considered a rigid body in the simulation model, a clamp move is used here to bolt the fender to DCS points of BIW at the bolting position.
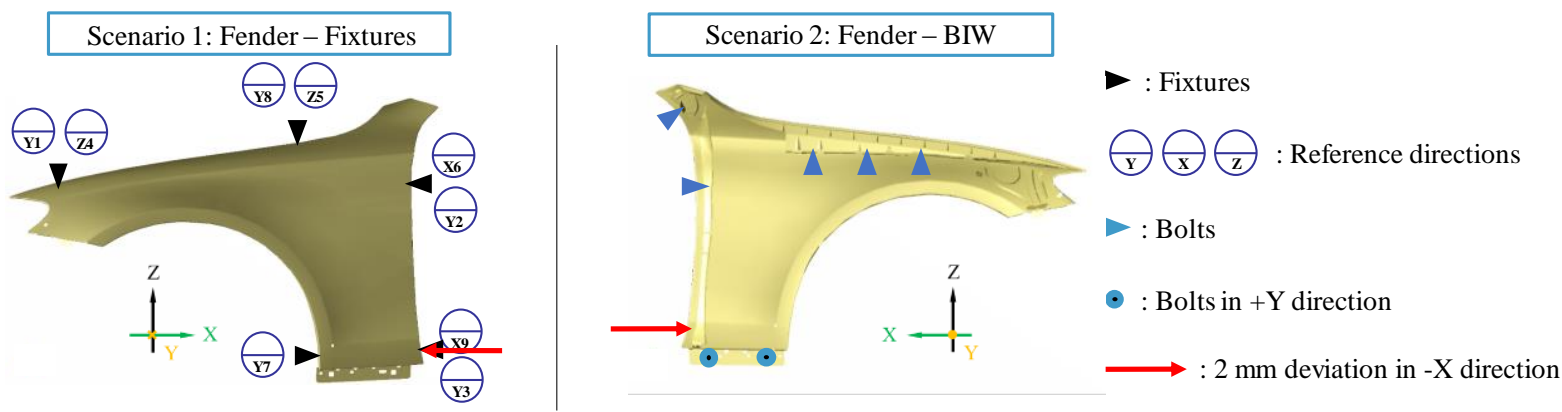

Figure 5: Scenario studies in two user-defined conditions 
In order to validate the simulation model after both stations, two scenarios are defined to implement the tolerance simulation. In the first scenario, the fender assembly is fixed after the first station at the black arrows on a measurement fixture. In the second scenario, the fender is bolted onto the BIW at the blue arrows (Figure 5).

The Best-Fit method, as described in the work of Kaestle (2010), is applied when mounting the fender to the BIW. The gap and the flushness between the fender and the bonnet are ensured firstly (in Y direction), the position of the fender is then adjusted in $\mathrm{Z}$ direction to block to the longitudinal beam, finally the rare flange of the fender is adjusted in X direction to ensure the gap to the front door. In the experience of real assembly process, $2 \mathrm{~mm}$ deviation in $-\mathrm{X}$ direction is constantly observed at the bottom of the fender (the red arrow), which results in large deviation in the front area. Therefore, in both simulation scenarios, a displacement of $2 \mathrm{~mm}$ in $-\mathrm{X}$ direction (the red arrow) is defined at the bottom of the fender by clamping the DCS point X9 to a target point that is $2 \mathrm{~mm}$ ahead in the $-\mathrm{X}$ direction. The simulation results of the measurement point (MP) in Figure 8 will be analyzed and evaluated in detail in the next sections.

\subsection{Scenario 1: Fender in the measurement condition}

The fender assembly is aligned on the measurement fixture by closing the clamps shown in scenario 1 , Figure 5. In 3DCS, a clamp move with "soft 1DOF" is applied for the fixture closing process, which only constrains specified degrees of freedom at the fixture locations. To verify the deviation in the front area that is mentioned in section 3.1, the statistical simulation results for the deviation in the $\mathrm{Y}$ direction are obtained for the measurement point (MP) and recorded in Figure 6. It indicates that when applying $2 \mathrm{~mm}$ displacement in -X direction at the bottom of the fender, MP has $3.26 \mathrm{~mm}$ deformation in -Y direction. After tolerance simulation, it has a mean shift of $0.34 \mathrm{~mm}$ in $\mathrm{Y}$ direction because of the tolerance influences. The deviation range of MP in $\mathrm{Y}$ direction is $2.67 \mathrm{~mm}$, which may be controlled by applying an extra fixture at that position.

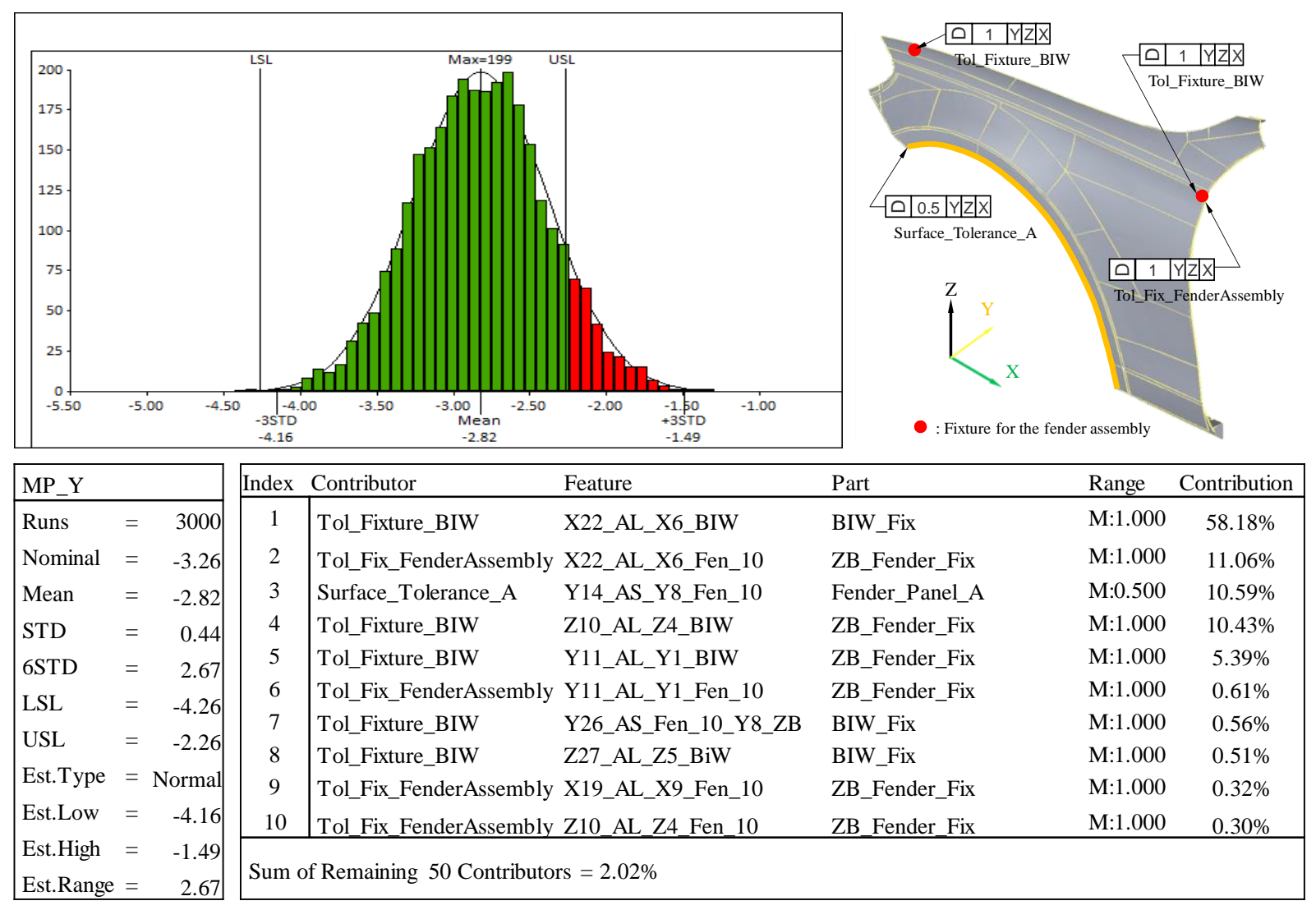

Figure 6: Fixture conditions of the fender in scenario 1

A sensitivity analysis report is generated by 3DCS after simulation. The contributors to the deviation distribution are also listed in Figure 6. The surface profile tolerances of the fixture X6 (red spot) has the largest influence on the deviation of the MP in Y direction. The surface profile tolerance of the 
fixture $\mathrm{X} 6$ and $\mathrm{Z} 4$ in the previous joint station as well as the surface profile tolerance in the front area of the panel A (orange area) also have an influence of over $10 \%$ on the deviation of the MP in Y direction.

\subsection{Scenario 2: Fender in the car condition}

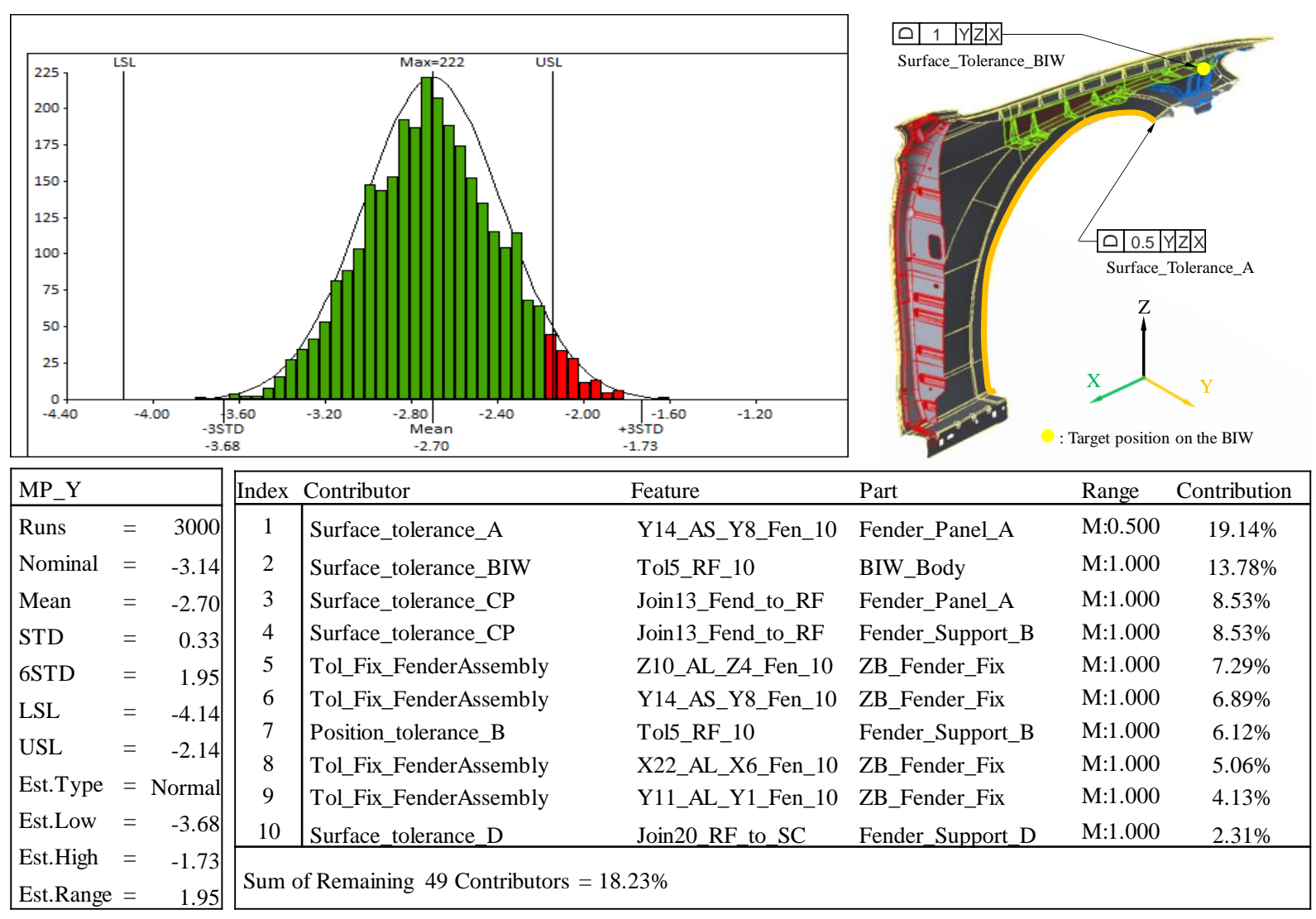

Figure 7: Fixture conditions of the fender in scenario 2

In this scenario, the fender is bolted at the position shown in Figure 4, which is the same as in the car condition. In this simulation model, the non-linear effect caused by bolting process is not considered, so the bolt joint is simulated by constraining DOFs at the bolted positions. Therefore, a clamp move with "hard 3DOF" is applied to simulate the bolting process. The statistical simulation results for MP in Y direction are recorded in Figure 7. Compared to the simulation results of scenario 1, the nominal value of the deviation of MP in Y direction is $3.14 \mathrm{~mm}$. It implies that, when the fender is bolted on the BIW with $2 \mathrm{~mm}$ displacement at the bottom, the front area will have a larger deviation from its design position. The mean value, the standard deviation and the deviation range of MP in Y direction decrease from Scenario 1 because the fender assembly is bolted in this scenario, which allows less flexibility than in Scenario 1. Additionally, according to the sensitivity analysis table, the surface profile tolerance in the front area of the panel A (orange area) and the surface profile tolerance of the target position on BIW (yellow spot) have the largest influence on the deviation of MP in Y direction.

\section{VALIDATION WITH PHOTOGRAMMETRY MEASUREMENT DATA}

To validate the simulation model, a real fender assembly is measured in the production line (Figure 8). Measurements with photogrammetry is used to measure the deviation. The measurement results are obtained in all $\mathrm{X}, \mathrm{Y}$ and $\mathrm{Z}$ directions for the user-defined measurement points. In the same manner as the simulation results, the front area has larger deformation after applying $2 \mathrm{~mm}$ displacement at the bottom of the fender. The deviation values of the highlighted position on the fender are compared to the simulation results of the measurement point MP. 

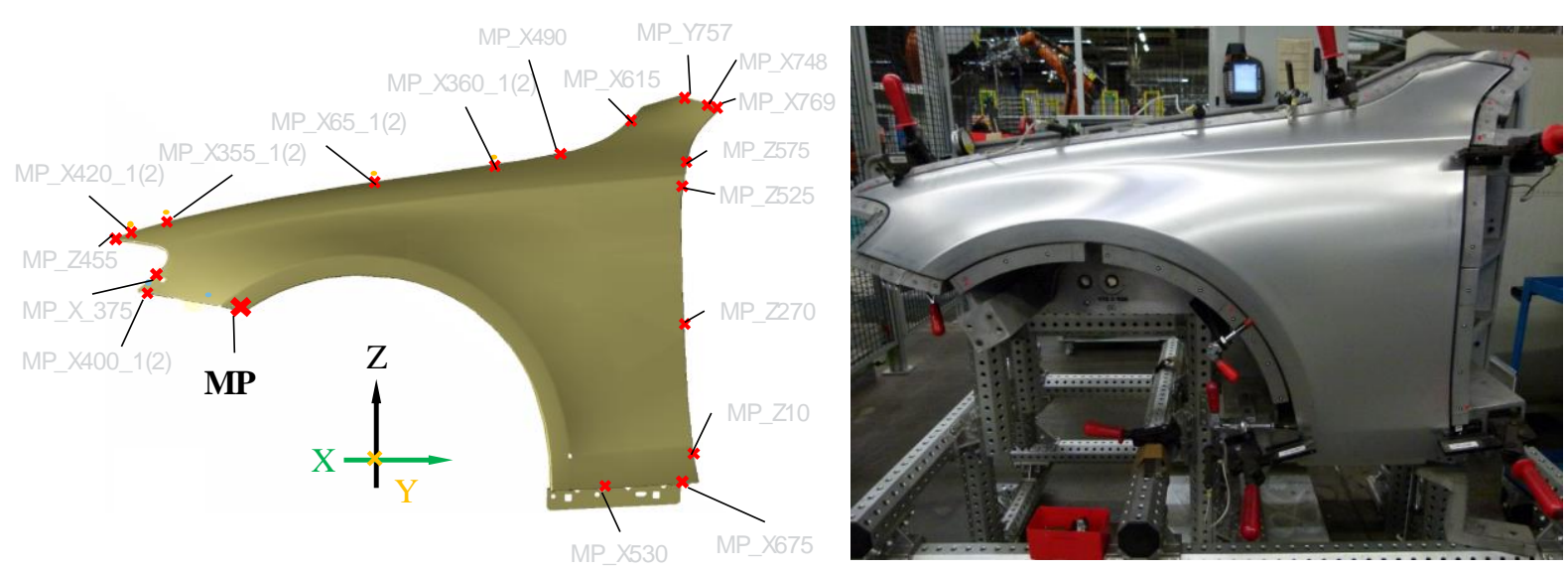

Figure 8: Measurement fixture and the measurement points on the fender

Table 1 and Table 2 are the comparison between the simulation results and the measurement results for MP for both Scenario 1 and Scenario 2. The displacement at X9 denotes the deviation at the bottom of the fender. The displacement in real measurement is not exact $2 \mathrm{~mm}$ in -X direction because of the manual operation error. The nominal value implies the influence of the assembly process on the deviation of the measurement point. The Mean value denotes the deviation that most probably occurs in the simulation after considering the tolerances. Moreover, all possible deviation values of the measurement point varies within the deviation range. Therefore, the nominal values as well as the mean values of the simulation results are compared with the deformation values in real measurement. The color mapping display to the right of the tables was generated in 3DCS and denotes the deformation of the simulation model. Generally, the simulation model of the fender in both scenarios has a similar deformation tendency after applying the $2 \mathrm{~mm}$ displacement at the same position. Large deformation is observed in the front area of the fender, which is consistent with the phenomenon that occurs in the real production process.

Specifically for the results of MP, the nominal values of the simulation model imply good consistency with the measurement results of the real fender assembly, which demonstrates the feasibility of the proposed modeling approach for the joint and assembly process for the fender-BIW system.

\section{Table 1: Validation of the simulation model in Scenario 1}

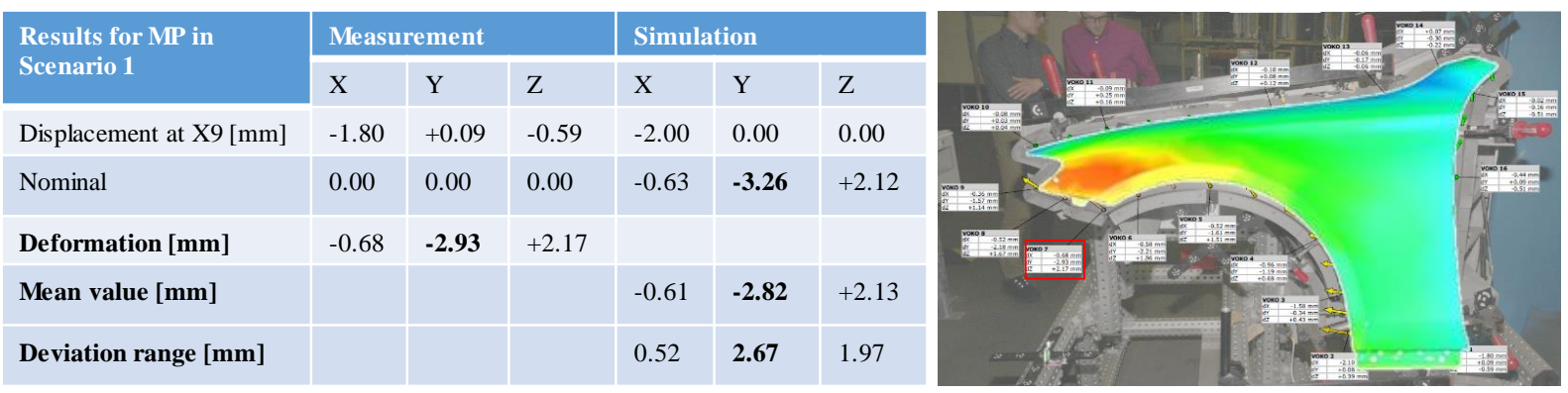

Table 2: Validation of the simulation model in Scenario 2

\begin{tabular}{|l|l|l|l|l|l|l|l|}
\hline $\begin{array}{l}\text { Results for MIP in } \\
\text { Scenario 2 }\end{array}$ & \multicolumn{3}{|l|}{ Measurement } & \multicolumn{3}{l|}{ Simulation } \\
\cline { 2 - 8 } & $\mathrm{X}$ & $\mathrm{Y}$ & $\mathrm{Z}$ & $\mathrm{X}$ & $\mathrm{Y}$ & $\mathrm{Z}$ \\
\hline Displacement at X9 [mm] & -1.97 & +0.11 & -0.25 & -2.00 & 0.00 & 0.00 \\
\hline Nominal & 0.00 & 0.00 & 0.00 & -0.58 & $\mathbf{- 3 . 1 4}$ & +2.45 \\
\hline Deformation [mm] & -0.64 & $\mathbf{- 3 . 2 4}$ & +2.72 & & & \\
\hline Mean value [mm] & & & & -0.56 & $\mathbf{- 2 . 7 0}$ & +2.46 \\
\hline Deviation range [mm] & & & & 0.28 & $\mathbf{1 . 9 5}$ & 1.18 \\
\hline
\end{tabular}


The measurement results of the real fender assembly deviate from the mean values of the simulation results but are still within the deviation range of the MP. Deviation between the simulation results and the measurement data can be affected by the following reasons:

- The non-linear effects exist in the real system: Linear deviations are calculated in the simulation model, so the non-linear behavior of the glue between parts as well as the plastic deformation of the hemmed flange in the hemming process, are not considered;

- Gravity of the fender assembly is not considered in the simulation model;

- Assumption that the BIW and fixtures are rigid parts;

- Operation errors during the real measurements.

In further studies, these factors can be reduced by optimizing the simulation model and using more advanced measurement methods. The possible optimizations of the simulation model are proposed in the next section.

\section{CONCLUSIONS}

This paper proposes an approach to implement tolerance simulation for a compliant assembly, which includes manufacturing processes such as clinching, bolting and hemming. The fender-BIW system is modeled as a compliant-rigid system and the simulation model is applied to two production scenarios. The deviation of the fender from its design position is measured in the tolerance simulation as well as in the real production line using photogrammetry. When applying $2 \mathrm{~mm}$ displacement in $-\mathrm{X}$ direction at the bottom of the fender, large deformation is observed in both simulation and real measurement. This demonstrates the efficacy of using simulation in early production as opposed to prototyping or other methods of design by showing the strong correlation between simulation results and as-built products. Because this approach is applied to analyze the tolerance of compliant parts considering the manufacturing process, it can also be adapted to other industrial scenarios where the deviation of assembly is affected by manufacturing processes. For example, a comparative study of Wang (2016) may be implemented to simulate the thin-walled C-section composite beam (TC2B) in an aircraft, the influence of the manufacturing sequence on the final deviation can be investigated by adjusting the compliant moves in the simulation model.

To bring the simulation results even closer to reality, a number of changes can be integrated into the approach:

1. The process containing plastic deformation can be simulated using assembly simulation, which involves non-linear effects as well as temperature effects.

2. A simulation system containing more compliant parts can be analyzed.

3. According to the concept of Litwa et al. (2015), an automatic integration process of points from CAD model to 3DCS could be realized by programming to increase the modeling efficiency.

\section{REFERENCES}

Camelio, J.A. and Hu, S.J. (2003), "Modeling Variation Propagation of Multi-Station Assembly Systems With Compliant Parts", Journal of Mechanical Design, Vol. 125 No. 4, pp. 673-681.

Corrado, A. and Polini, W. (2017), "A comprehensive study of analysis methods for rigid parts with manufacturing signatures and operating conditions", Journal of Advanced Mechanical Design, System, and Manufacturing, Vol. 11 No. 2, pp. JAMDSM0017-JAMDSM0017. https://doi.org/10.1299/jamdsm.2017jamdsm0017.

Schleich, B. and Wartzack, S. (2016), “A Quantitative Comparison of Tolerance Analysis Approaches for Rigid Mechanical Assemblies”, Procedia CIRP, Vol. 43, pp. 172-177. https://doi.org/10.1016/j.procir.2016.02.013.

Liu, S.C. and Hu, S.J. (1997), "Variation simulation for deformable sheet metal assemblies using finite element methods", Journal of manufacturing science and engineering, Vol. 119 No. 3, pp. 368-374.

Chang, M. and Gossard, D.C. (1997), "Modeling the assembly of compliant, non-ideal parts", Computer-Aided Design, Vol. 29 No. 10, pp. 701-708.

Moos, S. and Vezzetti, E. (2012), "Compliant assembly tolerance analysis: guidelines to formalize the resistance spot welding plasticity effects", The International Journal of Advanced Manufacturing Technology, Vol. 61 No. 5, pp. 503-518. https://doi.org/10.1007/s00170-011-3729-0.

Bohn, M. and Hetsch, K. (2013), Toleranzmanagement im Automobilbau, Carl Hanser Verlag GmbH Co KG, München. 
Ding, Y., Ceglarek, D. and Shi, J. (2000), "Modeling and diagnosis of multistage manufacturing processes: part I: state space model", Proceedings of the 2000 Japan/USA symposium on flexible automation, pp. 23-26.

Yan, H., Wu, X. and Yang, J. (2015), “Application of Monte Carlo Method in Tolerance Analysis", Procedia CIRP, Vol. 27, pp. 281-285. https://doi.org/10.1016/j.procir.2015.04.079.

Gerbino, S., Patalano, S. and Franciosa, P. "Statistical variation analysis of multi-station compliant assemblies based on sensitivity matrix”, International Journal of Computer Applications in Technology, Vol. 33 No. 1, pp. 12-23.

Franciosa, P. (2009), Modeling and simulation of variational rigid and compliant assembly for tolerance analysis, Doctoral dissertation, Ph. D Thesis, The University Naples Federico II, Italia.

Chen, W., Jin, S. and Lai, X. (2014), "Tolerance Optimization Considerations Applied to the Sheet Metal Compliant Assembly", Computer-Aided Design and Applications, Vol. 11 No. 1, pp. 68-76. https://doi.org/10.1080/16864360.2014.914413.

Wang, H., Zhang, S. and Yu, J. (2018), "Computer Aided Tolerancing of Composite Elevator Assembly Involving Clamping Forces Coordination”, Procedia CIRP, Vol. 75, pp. 256-260. https://doi.org/10.1016/j.procir.2018.04.057.

Barbero, B.R., Aragón, A.C. and Pedrosa, C.M. (2015), "Validation of a tolerance analysis simulation procedure in assemblies", The International Journal of Advanced Manufacturing Technology, Vol. 76 No. 5-8, pp. 1297-1310. https://doi.org/10.1007/s00170-014-6229-1.

Beckmann, A., Bohn, M. and Gust, P. (2015), “Tolerance Simulation in the Assembling Process based on Experimental Data from Series Production”, Procedia CIRP, Vol. 27, pp. 35-40. https://doi.org/10.1016/j.procir.2015.04.040.

Camelio, J.A., Hu, S.J. and Marin, S.P. (2004), “Compliant Assembly Variation Analysis Using Component Geometric Covariance”, Journal of Manufacturing Science and Engineering, Vol. 126 No. 2, pp. 355-360.

Yan, Y. (2019), Neue Systematik mit erweiterten Definitionen der Bezüge und Tolerierungen für Geometrieelemente, Doctoral dissertation, Ph. D Thesis, University of Stuttgart, Germany.

Kaestle, C. (2016), Simulationsmethode zur Beurteilung der Maßhaltigkeit von rollgefalzten Karosseriebaugruppen im Zusammenbau, Institut für Umformtechnik, Stuttgart, Stuttgart. http://dx.doi.org/10.18419/opus-9111.

Wang, H. and Liu, J. (2016), "Tolerance simulation of thin-wall C-section composite beam assembling with small displacement torsor model", Procedia CIRP, Vol. 43, pp. 274-279. https://doi.org/10.1016/j.procir.2016.02.015.

Litwa, F., Gottwald, M., Bohn, M., Klinger, J.F., Walter, M., Wartzack, S. and Vielhaber, M. (2015), "Automated point-based tolerance analysis model creation for sheet metal parts", Procedia CIRP, Vol. 27, pp. 65-70. https://doi.org/10.1016/j.procir.2015.04.045.

Dimensional Control System (2008), 3DCS Compliant Modeler, Dimension Engineering News.

Dimensional Control System (2010), Compliant Modeling, Dimensional Engineering News.

Dimensional Control System (2016), Compliant Move Toolbox, Dimensional Engineering News. 